Session 3557

\title{
The Virtual Industrial System: A Tool for Learning Production Planning Concepts and Techniques
}

\author{
Uanny M. Brens Garcia, Douglas A. Bodner, Spyros A. Reveliotis, \\ Chen Zhou, Jane C. Ammons, Marc Goetschalckx, T. Govindaraj, \\ Paul M. Griffin, Leon F. McGinnis and Gunter P. Sharp \\ Keck Virtual Factory Lab · School of Industrial and Systems Engineering \\ Georgia Institute of Technology
}

\section{Introduction}

Recent advances in technology have enabled greater use of computational tools within the undergraduate engineering curriculum, changing the way in which learning takes place. In particular, learning systems and tools can be designed to promote active learning and engineering experimentation, to allow students to manipulate realistic case study data rather than toy examples, and to incorporate visualization to aid in learning.

In this paper, we discuss efforts at Georgia Tech to create such web-based learning systems for courses in manufacturing and logistics within the undergraduate Industrial Engineering curriculum. The objective is to create a generic software system, called the Virtual Industrial System (VIS), and associated learning modules that focus on particular concepts to be taught. The learning modules consist of lab exercises with specific learning objectives and case study situations and data. The VIS is intended to provide modeling and analysis tools, a common reference model and database structure for case study data, a generic protocol for accessing and manipulating data, and a user interface with a common look and feel across different learning modules. The goal is to help students learn by facilitating their exploration of data, experimentation with and discovery of important factors in design and operation, and gaining insight through comparing alternative solutions.

We describe our progress and experience to date in prototyping and deploying modules, with a focus on a module in the area of production planning. The remainder of the paper is organized as follows. Section 2 reviews the literature in this area and motivates the VIS. In Section 3, we describe the VIS concept. Section 4 presents a specific VIS learning system in production planning. Section 5 discusses our deployment and assessment results to date. We conclude with plans for future work and dissemination of the VIS.

\section{Motivation and Background}

Improving student learning has motivated new approaches to teaching in the engineering curriculum. This has led to innovative hands-on and project-based courses, and to the use of educational technology. While an exhaustive listing of new teaching approaches and techniques is beyond the scope of this paper, several example efforts can be found in the literature ${ }^{1-4}$. On- 
line "tutoring systems" and monitoring systems represent one approach. These systems typically allow students to be aware of their class performance at all times. They can provide class notes and automatic grading of homework and quizzes. In addition, they help students organize their work and help make them aware of their standing in a class ${ }^{5,6}$.

There are numerous examples of case-based learning, online lectures, distance-learning courses, and industry-sponsored projects, whose aims are to increase student interest in learning. One trend is the concept of active learning, which engages and involves the student. Active learning can be facilitated significantly by technology, which for example makes it possible to recreate realistic case study situations for learning. Such software-based learning systems can let students make engineering decisions in a simulated system and see the outcomes of those decisions, without disrupting a real industrial environment ${ }^{2}$.

The growth of communications technology has provided additional opportunities to improve education. Commercial software applications, such as WebCT ${ }^{\mathrm{TM}}$, allow live e-conferences, web broadcasts and online forums, and video conferencing allows interactive distance teaching. Web-based instruction can be a medium for information retrieval and personal interactions among tutors and fellow students, and instructional methods such as reading multimedia, using interactive computer instruction and performing other research tasks makes the learning experience more active ${ }^{7}$. Educational institutions are investing significant resources and intellectual effort to provide robust computer hardware and software systems for interactive

learning ${ }^{2}$. In addition, schools encourage and in some cases require students to have a computer. With such effort being expended to develop new teaching methods and tools, it is important to be able to assess their effectiveness in terms of improving learning outcomes ${ }^{1,3,8}$. Techniques for assessment include transcript analysis, outcome analysis, student surveys and interviews, and focus groups.

To date, there are examples of interactive computational environments that promote learning of engineering concepts ${ }^{9}$. The majority of interactive tools, however, mainly contain "point and click" hypertext, hypermedia, and graphics. They present theory and examples so that users can view materials and navigate at their own pace. Siegel and Kirkley point out that while navigating through links and seeing pages can be "an absorbing experience in an ever-expanding, ever changing, information-rich environment," improved interactivity and more powerful instructional tools are needed to motivate and develop the problem solving abilities and research skills needed by students ${ }^{4}$. Our efforts are aimed at this need.

\section{The Virtual Industrial System}

To address the challenges and opportunities presented by applying new technology in the classroom, we are developing a set of web-based learning systems for undergraduate courses in manufacturing and logistics within Industrial Engineering. This set of systems is embodied in the Virtual Industrial System, which provides a generic software framework for the learning systems. As envisioned, the VIS provides a platform to teach students about the application of engineering methodology to real world problems using computational models. In doing so, the VIS exposes the students to large datasets and realistic problems, the kind that they will find in their careers after graduation. The models in the VIS must be designed so that they are 
configurable, to enable exploration of data and experimentation with alternative solutions. Finally, our goal is to facilitate active learning and motivate the learning of methodology through case study problems.

A specific learning system within the VIS is organized into what we call a course module, which is a set of curriculum materials and computational models that can be used by an instructor, for example, as an on-line lab assignment. A course module focuses on a particular concept or set of concepts to be taught. For example, a course module may focus on forecasting, or facility design, or production planning. To accomplish the goal of teaching these concepts, a course module consists of a standard set of components available to the instructor:

- a set of learning objectives that the course module is designed to achieve;

- a case study that describes a realistic problem from industry and includes realistic data that the student must analyze to solve the problem;

- an assignment template that accompanies the case study and describes the specific deliverables and other information needed for the students to perform the assignment;

- the web-based VIS tools, which incorporate the case study data and the set of computational tools needed to analyze the data and develop a solution to the problem;

- user manuals for the VIS system;

- a set of assessment tools that are tied to the learning objectives and are designed to test the effectiveness of learning; and

- $\quad$ reference material that may be used by the instructor as lecture material or as further reading for students.

As shown in Figure 1, the generic VIS contains four fundamental features:

- a data repository that provides a generic database structure for the case study data across different course modules;

- a common user interface that reduces the amount of time needed to learn how to use different course modules

- an integrated set of modeling tools that can be used by each course module; and

- a protocol for accessing and manipulating data, and for storing student solutions (as well as partial solutions), to be provided by application server software.

The database structure for the case study data is based on a reference model of manufacturing and logistics systems that has been under development at Georgia Tech ${ }^{10}$. This reference model is designed to provide a common structure for data across the different course modules for several reasons: (i) to aid in development of the learning systems, (ii) to provide students with a more unified way of conceptualizing manufacturing and logistics systems, and (iii) to promote consistent data representations for use in different tools and across different models. It provides representation for physical system elements (e.g., machines, transporters) and logical system elements (e.g., decision-making controllers, process sequences, bills of materials, etc.). 


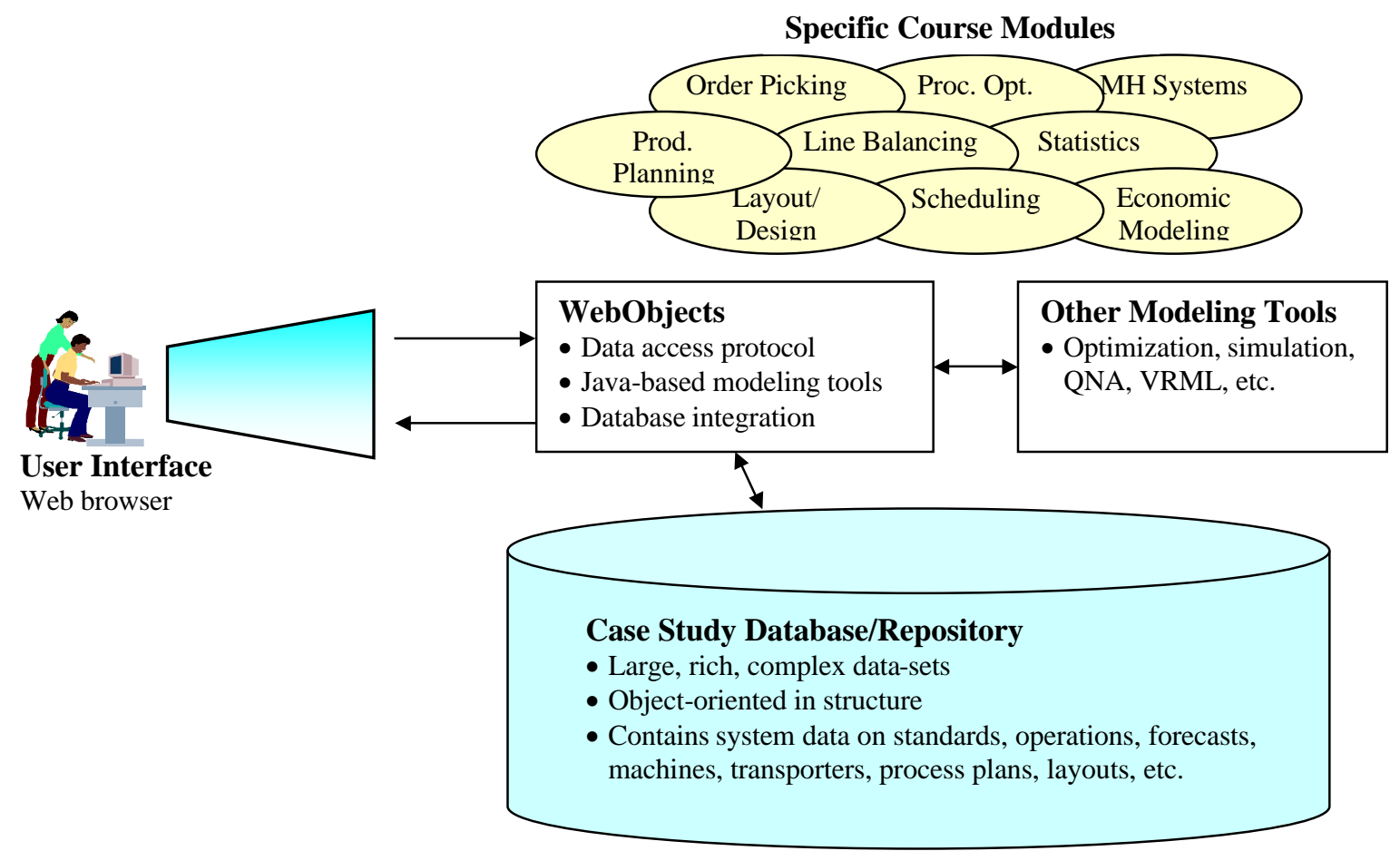

Figure 1. Generic VIS software architecture with associated course modules

For the data access protocol, we are using WebObjects ${ }^{\mathrm{TM}}$ from Apple Computer as the application server for the web-based VIS. WebObjects has powerful capabilities in terms of database connectivity, which is critical to the VIS. Certainly, development of web-based application is time-consuming. As a result, we have developed and deployed spreadsheet versions of several of the course modules to prototype the concepts and test their usability. To illustrate a specific instantiation of the VIS, the remainder of the paper focuses on a particular course module in production planning. We discuss the generic module, the use of a spreadsheet version of this module, and a web-based module that is under development.

\section{The Production Planning Module}

A critical component of Industrial Engineering education involves the concepts behind production planning, in which engineers determine a schedule of materials to release into a production system so that the finished goods are available to meet customer demand in specific time periods. The engineer must take production process lead-times into account, as well as capacity constraints (i.e., a limited set of processing equipment). Typically, the goal is to meet demand at minimum cost.

\subsection{The Generic Module}

The course module is designed to assist learning the intricacies of the production planning problem. A number of interacting issues must be considered in solving this problem, including costs, capacity, demand, lead times and product shelf life. Table 1 defines the generic terms and notation used in the production planning problem and module. 
Table 1. Terminology

\begin{tabular}{|c|c|}
\hline Term & Description \\
\hline $\begin{array}{l}\text { Product }(i= \\
1 \ldots m)\end{array}$ & Product type(s) produced by the system. \\
\hline $\begin{array}{l}\text { Period }(j= \\
1 \ldots n)\end{array}$ & $\begin{array}{l}\text { Discrete unit of time into which the production time horizon is partitioned. A period may } \\
\text { be a week within a time horizon of three months, for example. }\end{array}$ \\
\hline Demand $\left(d_{i j}\right)$ & Forecasted customer demand for product $i$ in period $j$. \\
\hline $\begin{array}{l}\text { Schedule } \\
\text { receipts }\left(S R C_{i j}\right)\end{array}$ & $\begin{array}{l}\text { Amount of product } i \text { already scheduled to be finished in period } j \text {. This represents an } \\
\text { initial condition, since at startup there are likely to be batches of product already in } \\
\text { production, ready for release at some known future period. }\end{array}$ \\
\hline $\begin{array}{l}\text { Inventory } \\
\text { position }\left(I_{i j}\right)\end{array}$ & $\begin{array}{l}\text { Amount of finished product } i \text { in inventory during period } j . I_{i j} \text { shows amount of product is } \\
\text { available, after subtracting spoilage and satisfied demand for period } j \text {, and adding } \\
\text { completed production. }\end{array}$ \\
\hline $\begin{array}{l}\text { Net requirement } \\
\left(N R_{i j}\right)\end{array}$ & $\begin{array}{l}\text { Amount of unsatisfied demand of product } i \text { in period } j . \text { If } N R_{i j}=0 \text {, demand is met by the } \\
\text { production plan. If } N R_{i j}>0 \text {, the unmet demand results in lost sales. In this case, the } \\
\text { student should infer that the production plan needs to be changed to meet demand. }\end{array}$ \\
\hline $\begin{array}{l}\text { Schedule release } \\
\left(S R L_{i j}\right)\end{array}$ & Amount of raw materials for product $i$ to release into the system in period $j$. \\
\hline Capacity $(C A P)$ & Total capacity of plant, summed over all equipment. \\
\hline $\begin{array}{l}\text { Capacity used } \\
\left(C A P U_{j}\right)\end{array}$ & $\begin{array}{l}\text { Equipment capacity in use in period } j \text {, including discrete effects. These occur when a } \\
\text { piece of equipment is counted as fully utilized, even the amount of product it is } \\
\text { processing is less than its capacity, due to its having to finish that product batch before } \\
\text { starting a new batch. }\end{array}$ \\
\hline
\end{tabular}

The module serves as a tool for students to understand these interactions in the context of multiproduct production and cost minimization, where costs include raw materials costs, holding costs, sequence-dependent setup costs, inventory costs and spoilage costs. For example, one strategy is to produce large batches of product in a few periods, so as to result in fewer setup costs. This strategy uses inventory buildup from a finished batch to satisfy demand for several periods after the batch is finished. As a consequence, though, this strategy incurs inventory costs, and it may incur spoilage costs. In addition, the capacity constraints generally bound the size of the batches. Another approach is to produce just enough of each product in each period to satisfy demand. This results in no inventory or spoilage costs, but it incurs a setup cost for each period. Hence, there is a fundamental trade-off between setup cost versus inventory and spoilage costs. Using the module, a student can explore this trade-off, experiment with different solutions, and learn how to determine overall minimum cost.

It is important to note that this problem can be solved optimally by formulating it as a mixed integer programming (MIP) problem ${ }^{11}$. This approach is too advanced, however, for the level of our target student population, which typically does not yet have exposure to integer programming methodology. Therefore, we have adopted the experimental approach to learning, in which students can define a production plan and then modify their plan to achieve improved cost. The students manipulate the scheduled release of raw materials into the system $\left(S R L_{i j}\right)$, and the VIS computes the resulting production schedule, tells whether or not it is feasible (i.e., whether the capacity constraints are violated), and returns the cost. One of our goals is to use 
realistic datasets that cannot be solved by inspection or easy computation, motivating students to explore the data and experiment with different solutions. Through such experimentation, students learn about trade-offs and interactions between key problem elements, and (we hope) become motivated to learn more advanced methods such as MIP to solve these types of problems. Our specific learning objectives for this module include the following:

- to help the student develop an intuitive understanding of production planning in a manufacturing environment with multiple-product demand and with lead time, production capacity and shelf-life constraints;

- to familiarize the student with (i) the underlying trade-offs among inventory holding, frequent production setups, partially loaded batches, shortages/lost sales and spoilage, and (ii) the modeling of these trade-offs through the introduction of an appropriate cost structure;

- to motivate to the student the need for the development and use of more sophisticated computational techniques and algorithms for the solution of production planning problems.

The case study currently in use involves a microbrewery production system that produces several types of beer, many of which have seasonal demands. This demand pattern complicates the production planning. The beers are produced in fermentors, and each beer has a fixed lead time (process time in a fermentor) and shelf life. The plant has a set number of fermentors with a fixed capacity. The case study data also include demand for each product in each period and covers five product types over a period of 26 weeks.

\subsection{Spreadsheet Prototype}

Our first implementation of this module is in spreadsheet form using Microsoft ${ }^{\circledR}$ Excel. The spreadsheet contains three worksheets. The Master Production Scheduling (MPS) worksheet provides an interface for user input data entry (scheduled releases) and for production schedule and inventory level results, including feasibility. The Demand and Inventory Position worksheet provides a chart showing demand and inventory levels in each period for each product. The inventory levels change based on the user inputs. Finally, the Lost Sales worksheet charts lost sales (i.e., when production does not satisfy demand in a period) and computes a lost sale cost. The lost sales also depend on the user inputs.

Learning can occur through several avenues in the module. First, the students can experiment with different types of production release schedules to see which yield lower costs (e.g., frequent small releases vs. infrequent large releases). Since the tool handles the tedious computations and presents the results of a schedule quickly, it allows the student to try many different scheduling strategies in a reasonable amount of time. Second, if the release schedule is infeasible (i.e., over capacity at some point in time), the spreadsheet flags the infeasibility, and the student can correct it. Through this feature, students learn the relationship between release dates, lead times and shelf lives, and capacity constraints. Finally, the graphs provided by the Inventory and Position worksheet and the Lost Sales worksheet give students a visual representation of the problem data and solutions, in addition to the tabular view given in the MPS worksheet. This helps students further learn production planning concepts.

Due to the limits of spreadsheet technology, the MPS worksheet is customized toward the microbrewery case study, which features a single-step production process and a single-level bill 
of materials (i.e., the list of items needed for a finished product). The spreadsheet incorporates complex formulas representing the interactions between problem elements so that the user does not need to access them.

\subsection{Web-Based Module}

We have developed a web-based module using the same microbrewery case study. The webbased module is implemented using WebObjects and uses a database representation for a more generic way of representing the problem data. Since students are familiar with a spreadsheet interface, the module retains an interface similar to the spreadsheet version's MPS worksheet. The user needs a Java ${ }^{\mathrm{TM}}$-enabled web browser in order to access the module.

In designing the web-based application, we have relied on the use case methodology of Fowler and Scott ${ }^{12}$. This methodology calls for the specification of typical user interactions and functionalities (i.e., use cases) to be supported by a software system, as input into the design process. For this application, there are two types of users: the instructor and the student, and hence two classes of use cases. In general, the instructor should be able to perform any function that a student can. The instructor needs additional functionality, such as entering and modifying problem data, retrieving student solutions, etc. Figure 2 illustrates a use case diagram for several student and instructor functions.

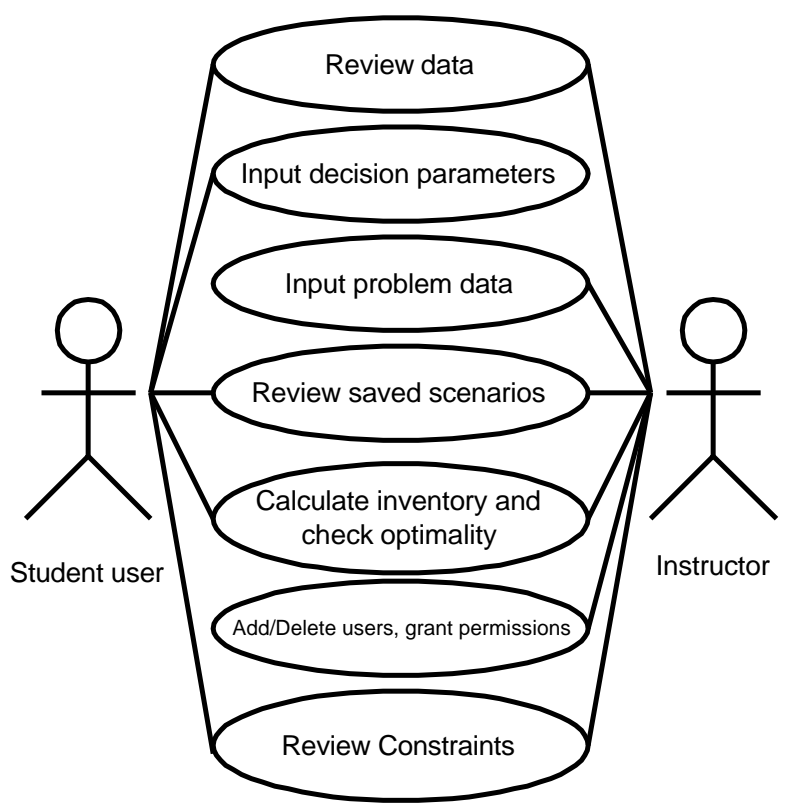

Figure 2. Example use case diagram

The module has a log-in page for use by instructors and students, into which a user name and password are entered. The WebObjects application grants access with the appropriate level. The students access the course module, experiment with different production plans to determine the "best," and then submit their solution. Students also can save work-in-process solutions, $\log$ out, and return to them later. The computations of the problem are implemented in Java within the WebObjects framework. These include generation of a schedule, determination of its feasibility 
and the computation of inventory levels. The cost computations are under development. The instructor updates problem data as needed (e.g., for offerings of the module in different terms) and can view all the user files, but cannot modify them. Students, of course, can view only their own files. Table 2 summarizes the access rights of students and instructors.

Table 2. User access

\begin{tabular}{|l|l|}
\hline \multicolumn{1}{|c|}{ Student } & \multicolumn{1}{|c|}{ Instructor } \\
\hline \hline Log into the module & Log into the module \\
Problem data: read access & Problem data: read/write access \\
Input fields with read/write access & User's database maintenance \\
Check feasibility of input & Check feasibility of model \\
Read inventory levels & Read/write inventory levels \\
Read demand & Read/write demand \\
Read/write input parameters (save and submit output) & Read user's saved model \\
\hline
\end{tabular}

The problem data are stored in a database that is accessed by WebObjects. The case study data are contained in three tables (product data, production plan data and plant data). A fourth table contains the user's solution. Table 3 shows the data fields of each table. It should be noted that the students can perform what-if analysis by changing data in the production plan table, to determine how these changes affect their production plan. This supports our efforts to let the students experiment and gain understanding of the interactions of these problem elements.

Table 3. Database tables

\begin{tabular}{|l|}
\hline 1. Product Data \\
\hline \hline Product name \\
Lead time \\
Shelf life \\
Product cost \\
Holding cost \\
Spoilage cost \\
\hline
\end{tabular}

\begin{tabular}{|l|}
\hline 2. Production Plan Data \\
\hline \hline Period ID \\
Product name \\
Demand \\
Inventory \\
Net requirements \\
Schedule receipt \\
Equipment needed \\
\hline
\end{tabular}

\begin{tabular}{|l|}
\hline 4. Solution Data \\
\hline \hline User name and/or ID \\
Schedule releases per \\
period and product \\
\\
\hline
\end{tabular}

In using the module, a student navigates through various pages to view problem data, make decisions, and determine the resulting schedule and its feasibility. Figure 3 summarizes this navigation.

\section{Deployment and Assessment}

To date, the spreadsheet version of the production planning module has been deployed during three semesters in the target junior-level course in the Industrial Engineering curriculum at Georgia Tech. This course, titled "Supply Chain Module: Manufacturing and Warehousing," covers design and operational issues in manufacturing and warehouse facilities. It is taken by approximately 300 students per year. The course module provides flexibility in the ways in which it can be used. Thus far, the instructors have assigned it as a homework project, in which three to four students collaborate to solve the problem, and then submit a report to the 


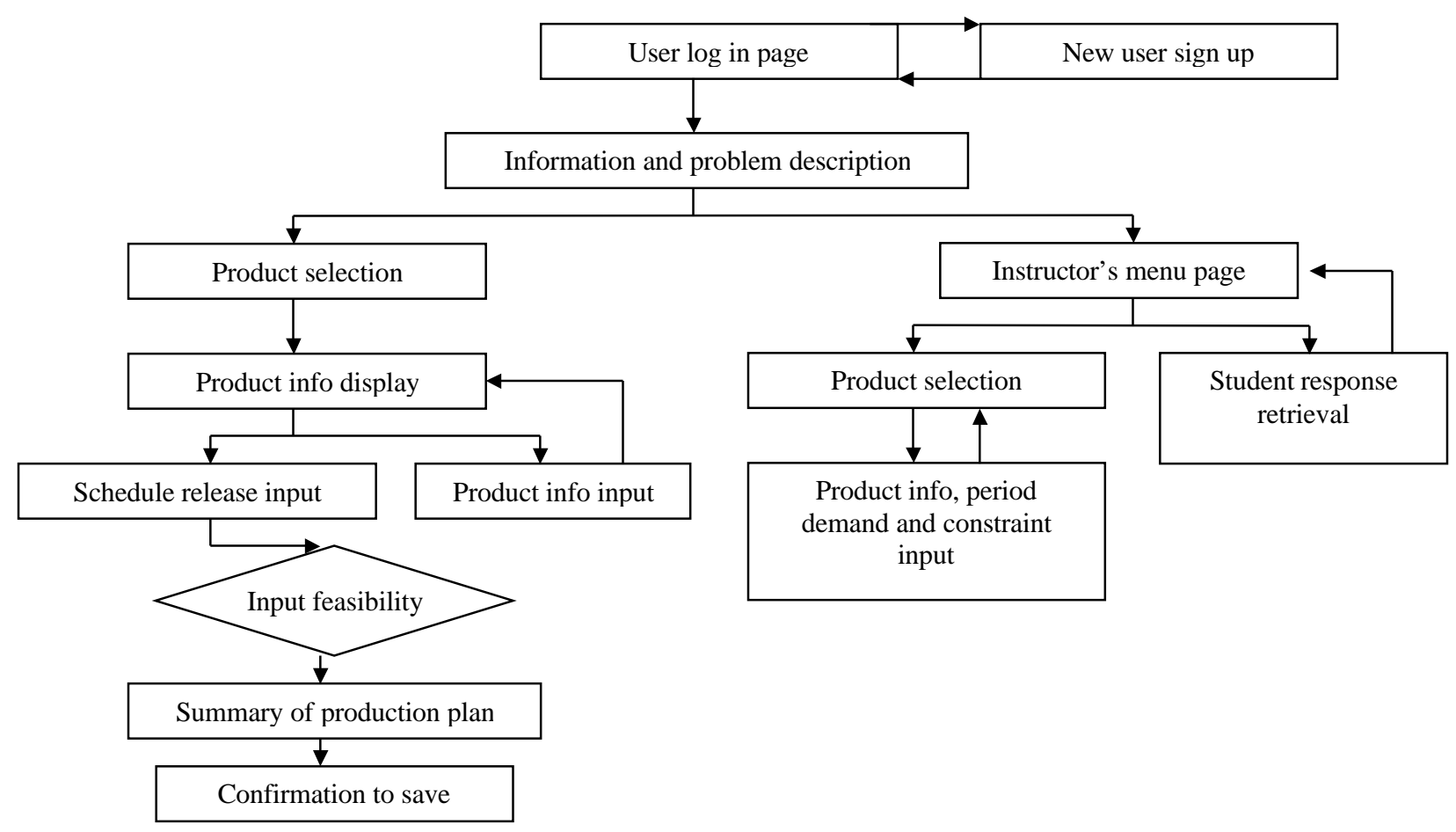

Figure 3. Flowchart of web-based module's interface

microbrewery management with their recommendations for a production plan. Through this approach, students learn how to work in teams, interpret engineering results for presentation to management, and write a technical report with appropriate structure and style, graphical aids and justification of results.

Our emphasis so far has been on formative assessment, in which we seek to understand how students feel toward the course module experience, rather than on a summative assessment that objectively measures how they learned from the experience. Insight from the formative assessment is useful, because it points out avenues of potential improvement in the module. We are developing summative assessment tools that link with the module's learning objectives.

After use of the module, a formative assessment questionnaire is given to the student to collect their feedback. The questionnaire contains a series of questions, and the students are asked to assign a value between 1 and 4 to represent their feelings in terms of answers to the questions. In this scheme, the value " 1 " represents "strongly agree"; the value " 2 " represents "agree"; the value " 3 " represents "disagree"; and the value " 4 " represents "strongly disagree." The questionnaire also contains a comments section asking for students' "most favorite" thing about using the module, "least favorite" thing, and one idea for improving the module. Table 4 summarizes quantitative results from the questionnaire over three semesters.

If we assume that an average value of 2.5 for any response represents indifference between agreement and disagreement, we can use hypothesis testing to determine whether there is any 
statistical significance that students agree or disagree with the statements in the questionnaire. Using a $t$-test at a level of significance of $\alpha=0.05$, we would reject the hypothesis that the students are indifferent in favor of the hypothesis that they agree with the statements in the last four lines of the table, over all three semesters. We interpret this to mean that the students feel, on average, that the module helped them learn the concepts stated in the table, and that they recommend its future use.

Table 4. Assessment results of the spreadsheet module

\begin{tabular}{|l|l|l|l|l|l|l|}
\hline \multicolumn{2}{|c|}{} & \multicolumn{2}{|c|}{$\begin{array}{c}\text { Sum } 2000 \\
(n=62)\end{array}$} & \multicolumn{2}{|c|}{$\begin{array}{c}\text { Fall 2000 } \\
(n=36)\end{array}$} & \multicolumn{2}{|c|}{$\begin{array}{c}\text { Spr 2001 } \\
(n=29)\end{array}$} \\
\hline Question & Avg & $\begin{array}{c}\text { Std } \\
\text { Dev }\end{array}$ & Avg & $\begin{array}{c}\text { Std } \\
\text { Dev }\end{array}$ & Avg & $\begin{array}{c}\text { Std } \\
\text { Dev }\end{array}$ \\
\hline \hline $\begin{array}{l}\text { After using the production planning module, it is still useful } \\
\text { to attend class lectures on production planning. }\end{array}$ & 1.85 & 0.57 & 1.69 & 0.52 & 1.97 & 0.57 \\
\hline $\begin{array}{l}\text { Using the production planning module helped me } \\
\text { understand class lectures. }\end{array}$ & 2.13 & 0.68 & 1.75 & 0.50 & 2.00 & 0.65 \\
\hline $\begin{array}{l}\text { I would have preferred traditional instruction followed by } \\
\text { homework exercises rather than using the production } \\
\text { planning module. }\end{array}$ & 2.44 & 0.74 & 2.83 & 0.70 & 2.62 & 0.78 \\
\hline $\begin{array}{l}\text { I had difficulties or problems using the Excel spreadsheets } \\
\text { and Excel program (this excludes difficulties you may have } \\
\text { had understanding or using the production planning } \\
\text { formulas and graphs). }\end{array}$ & 2.82 & 0.70 & 3.31 & 0.52 & 3.00 & 0.68 \\
\hline After using the module, I am confident that I understand: & & & & & & \\
\hline $\begin{array}{l}\text { a. Concept of backwards scheduling with finite processing } \\
\text { resources. }\end{array}$ & 2.24 & 0.64 & 1.97 & 0.65 & 2.14 & 0.58 \\
\hline$\quad$ b. How to minimize costs in production planning. & 2.13 & 0.66 & 1.86 & 0.59 & 2.28 & 0.53 \\
\hline $\begin{array}{l}\text { c. Effects of multiple products in the production planning } \\
\text { process. }\end{array}$ & 2.07 & 0.65 & 1.89 & 0.67 & 2.21 & 0.56 \\
\hline $\begin{array}{l}\text { I recommend that this module be used as an instruction aid } \\
\text { in future classes. }\end{array}$ & 1.84 & 0.64 & 1.69 & 0.58 & 2.10 & 0.56 \\
\hline
\end{tabular}

Similarly, we draw the conclusion that the students prefer to have lectures along with the module to help them understand concepts. This is reinforced by comments solicited in the comments section of the questionnaire. A common theme among many comments is that the students would have preferred more lecture time, as well as instructions on use of the spreadsheet. This implies that it may be necessary to provide at least some structure to student experimentation in engineering problem-solving. On the other hand, the student comments reveal benefits that they experienced from use of the module. These include the use of realistic data and problems to illustrate class lecture material, as well as access to a computational tool that allows easy manipulation of large datasets.

The current questionnaire does not provide open-ended input as an interview or focus group might. Also, it is important to note that this formative assessment addresses only how students feel. It does not provide an objective assessment of learning outcomes provided by the module, for example, as a pre-test and post-test on course material might. An objective assessment of 
learning outcomes is important, and it can be used in a feedback loop to provide inputs for improving teaching methods and tools ${ }^{13,14}$. Nevertheless, the formative assessment has provided useful information, as we work toward developing more objective assessment methods.

\section{Conclusion}

This paper has discussed the development of a computational learning system for the Industrial Engineering curriculum, with a focus on a module that covers production planning. In developing this system, our goal is to promote active learning and student experimentation with engineering problems using realistic case study datasets. The production planning problem provides a good illustration of our approach. The case study dataset is large enough that a student needs a computational tool for developing a production plan, and our experimental approach allows the students to explore and perform what-if analysis to learn about the interactions between problems elements.

A spreadsheet version of the module has been used in the curriculum. Our future work involves deploying the web-based module, which is scheduled for spring semester 2002. We are developing summative assessment tools in the form of pre-tests and post-tests that will be used to measure the effectiveness of the module in terms of student learning. It is important to have quantitative data and analysis to demonstrate the effect of a module on student learning.

Since the modules are in web-based form, they can be accessed from locations other than our campus. We are looking to develop partnerships with other universities to test the effectiveness of the VIS system and associated modules at schools other than Georgia Tech.

\section{Acknowledgments}

This work is funded in part by the National Science Foundation under grant no. DUE-9950301. The authors also would like to acknowledge support from the W. M. Keck Foundation and the Georgia Tech Research Corporation, as well as work done to implement the production planning spreadsheet by Mark Insalaco.

\section{Bibliography}

1. Renshaw, A. A., Reibel, J. H., Zukowski, C. A., Penn, K., McClintock, R. O., and Friedman, M. B. “An Assessment of On-Line Engineering Design Problem Presentation Strategies," IEEE Transactions on Education, Vol. 43, No. 2, pp. 83-91, 2000.

2. Park Woolf, B. "Intelligent Multimedia Tutoring Systems," Communications of the ACM, Vol. 39, No. 4, pp. 30-31, 1996.

3. Marr, M. J., Thomas, E. M., Been, M. R., Thomas, A., and Hume, R. M. "Development of Instructional Systems for Teaching an Electricity and Magnetism Course for Engineers," American Journal of Physics, Vol. 67, No. 9, pp. 789-802, 1999.

4. Siegel, M., and Kirkley, S. "Moving Toward the Digital Learning Environment: The Future of Web-Based Instruction," in Web-Based Instruction, Khan, B. H. (ed.), Englewood Cliffs, NJ: Education Technology Publications, pp. 263-270, 1997. 
5. Garcia-Beltran, A., Jaen, J., Martinez, R., and Criado, J. "WebTutor, a Self-Evaluation and Student Tracking System," Proceedings of the North American Web Developers Conference, October 1998.

6. Chen, G. D., Liu, C., Ou, K., and Lin, M. "Web Learning Portfolios: A Tool for Supporting Performance Awareness," Innovations in Education and Training International, Vol. 28, No. 1, 2001.

7. Bostock, S. J. "Designing Web-Based Instruction for Active Learning," in Web-Based Instruction, Khan B. H. (ed.), Englewood Cliffs, NJ: Education Technology Publications, pp. 225-230, 1997.

8. Thornton, R. K., and Sokoloff, D. R. “Assessing Student Learning of Newton's Law: The Force and Motion Conceptual Evaluation and the Evaluation of Active Learning Laboratory and Lecture Curricula," American Journal of Physics, Vol. 66, No. 4, pp 338-347, 1998.

9. Dessouky, M. M., Bailey, D. E., Verma, S., Adiga, S., Bekey, G. A., and Kazlauskas, E. J. “A Virtual Factory Teaching System in Support of Manufacturing Education," Journal of Engineering Education, Vol. 87, No. 4, pp. 459-467, 1998.

10. Khachatryan, M. R., Bodner, D. A., Ammons, J. C., Goetschalckx, M., Griffin, P. M., McGinnis, L. F., Reveliotis, S. A., Sharp, G. P., and Zhou, C. "Computational Learning Environments in IE Education," Proceedings of the 2002 Industrial Engineering Research Conference, to appear in 2002.

11. Nahmias, S. Production and Operations Analysis, Fourth Edition, McGraw-Hill, 2001.

12. Fowler, M., and Scott, K. UML Distilled, Second Edition, Reading, MA: Addison-Wesley, 2000.

13. Apostolou, B. "Education Research: Outcomes Assessment," Issues in Accounting Education, Vol. 14, No. 1, pp. 177-197, 1999.

14. Bolin, P. E. "What Is Taught? What Is Learned? How Do We Know?" Art Education, Vol. 52, No. 5, 1999.

Biographies

UANNY M. BRENS GARCIA received her Master's in Manufacturing Engineering from Rochester Institute of Technology and currently is a Ph.D. student in the School of Industrial and Systems Engineering at the Georgia Institute of Technology. She is a member of IIE and of the Committee of Industrial Students of PUCMM Dominican Republic. Her research interests are in the areas of manufacturing and education.

DOUGLAS A. BODNER is a research engineer in the School of Industrial and Systems Engineering at the Georgia Institute of Technology, where he manages the Keck Virtual Factory Lab. He is a member of IIE, INFORMS and IEEE. His research interests involve production systems and integrated modeling technologies.

SPYROS A. REVELIOTIS is an assistant professor in the School of Industrial and Systems Engineering at the Georgia Institute of Technology. He is a member of IEEE and IIE. His research addresses scheduling and analysis of discrete event systems and flexibly automated production systems.

CHEN ZHOU is an associate professor in the School of Industrial and Systems Engineering at the Georgia Institute of Technology. He is a member of IEEE and SME. His research focuses on manufacturing systems, facility layout, and automation.

JANE C. AMMONS is NSF ADVANCE Professor of Engineering in the College of Engineering and a professor the School of Industrial and Systems Engineering at the Georgia Institute of Technology. She is a member of IIE. Her interests include material flow systems, supply chain integration, reverse logistics, and environmentally conscious systems.

MARC GOETSCHALCKX is an associate professor in the School of Industrial and Systems Engineering at the Georgia Institute of Technology. He is a member of IIE and INFORMS. His interests include design of logistics systems, facilities and supply chain systems.

T. GOVINDARAJ is an associate professor in the School of Industrial and Systems Engineering at the Georgia Institute of Technology. He is a member of AAAS, ACM and IEEE. His research interests involve human-machine systems, information engineering and manufacturing systems.

Proceedings of the 2002 American Society for Engineering Education Annual Conference and Exposition Copyright $\odot$ 2002, American Society for Engineering Education 
PAUL M. GRIFFIN is an associate professor in the School of Industrial and Systems Engineering at the Georgia Institute of Technology. His research addresses problems in production and logistics systems and supply chain control and integration.

LEON F. MCGINNIS is Gwaltney Professor of Manufacturing Systems in the School of Industrial and Systems Engineering at the Georgia Institute of Technology, where he has been leading research in manufacturing logistics systems, applied computation and applied operations research since 1975. He is a Fellow of IIE.

GUNTER P. SHARP is an associate professor in the School of Industrial and Systems Engineering at the Georgia Institute of Technology. He is a member of INFORMS and WERC. His research interests include storage/retrieval and material flow systems and economic decision analysis. 